ARTICLE

DOI: $10.1038 / \mathrm{s} 41467-017-00351-8$

OPEN

\title{
Formation pathways of mesoporous silica nanoparticles with dodecagonal tiling
}

Yao Sun ${ }^{1}$ Kai Ma1, Teresa Kao ${ }^{1}$, Katherine A. Spoth², Hiroaki Sai (1) ${ }^{1,5}$, Duhan Zhang ${ }^{1}$, Lena F. Kourkoutis ${ }^{2,3}$, Veit Elser $^{4} \&$ Ulrich Wiesner ${ }^{1}$

Considerable progress in the fabrication of quasicrystals demonstrates that they can be realized in a broad range of materials. However, the development of chemistries enabling direct experimental observation of early quasicrystal growth pathways remains challenging. Here, we report the synthesis of four surfactant-directed mesoporous silica nanoparticle structures, including dodecagonal quasicrystalline nanoparticles, as a function of micelle pore expander concentration or stirring rate. We demonstrate that the early formation stages of dodecagonal quasicrystalline mesoporous silica nanoparticles can be preserved, where precise control of mesoporous silica nanoparticle size down to $<30 \mathrm{~nm}$ facilitates comparison between mesoporous silica nanoparticles and simulated single-particle growth trajectories beginning with a single tiling unit. Our results reveal details of the building block size distributions during early growth and how they promote quasicrystal formation. This work identifies simple synthetic parameters, such as stirring rate, that may be exploited to design other quasicrystal-forming self-assembly chemistries and processes.

\footnotetext{
${ }^{1}$ Department of Materials Science and Engineering, Cornell University, Ithaca, NY 14853, USA. ${ }^{2}$ School of Applied and Engineering Physics, Cornell University, Ithaca, NY 14853, USA. ${ }^{3}$ Kavli Institute at Cornell for Nanoscale Science, Ithaca, NY 14853, USA. ${ }^{4}$ Department of Physics, Cornell University, Ithaca, NY 14853, USA. ${ }^{5}$ Present address: Simpson Querrey Institute for Bionanotechnology, Northwestern University, Evanston, IL 60201, USA. Yao Sun, Kai $\mathrm{Ma}$ and Teresa Kao contributed equally to this work. Correspondence and requests for materials should be addressed to U.W. (email: ubw1@cornell.edu)
} 
uasicrystals exhibit highly ordered local structure but lack long-range translational periodicity, and permit symmetry operations that are forbidden in classical crystallography. Since the discovery of quasicrystalline order in metal alloys ${ }^{1}$, quasicrystals have been observed in a wide variety of materials, including thin films ${ }^{2}$, liquid crystals ${ }^{3}$, polymers ${ }^{4}$, colloids ${ }^{5}$, and mesoporous networks ${ }^{6}$, demonstrating that quasicrystallinity can be considered a universal form of ordering.

Advances in the identification of quasicrystalline materials have attracted widespread attention to the underlying mechanisms that govern quasicrystal formation. In self-assembled micellar systems, access to quasicrystal and quasicrystal approximant phases depends on building block geometry, controlled in recent experimental studies through molecular design of giant surfactant molecules $^{7,8}$ and asymmetric diblock copolymers ${ }^{9,10}$. These experimental findings are consistent with simulations of self-assembled systems, where building blocks designed with substantial shape polydispersity or patchy surfaces promote quasicrystalline order ${ }^{11,12}$. In metal alloys, studies of quasicrystal formation have verified theoretical predictions that quasicrystalline order is maintained during grain growth by a local error-andrepair type mechanism ${ }^{13}, 14$. Despite these successes, experimental investigations of early quasicrystal formation mechanisms remain challenging, largely due to a lack of chemistries that facilitate direct observation.

Mesoporous silica materials with pore sizes in the range between 2 and $50 \mathrm{~nm}$ have attracted widespread attention due to their precisely tunable macroscopic form, chemical functionality, and mesopore structure ${ }^{15}, 16$. There have been reports of mesoporous silica bulk materials ${ }^{15-18}$ and nanoparticles ${ }^{19-21}$ with a variety of pore structures, including hexagonal ${ }^{16}$, cubic $^{20}$, and quasicrystalline structures $6,20,22$. The formation of mesoporous silica is a result of silica condensation directed by molecular templates, such as surfactants or polymers ${ }^{16,23}$. This cooperative assembly process is driven by non-directional interactions between micelles and silica precursors, making mesoporous silica similar to systems of metal alloys, where non-directional bonding plays a key role in achieving the non-periodic bonding geometries required for quasicrystal formation.

Inspired by this analogy, we have synthesized surfactantdirected mesoporous silica nanoparticles (MSNs) with dodecagonal tiling and used them as a test bed for studying early formation mechanisms. While previous reports of quasicrystalline mesoporous silicas have used anionic surfactants 6,22 , we demonstrate that such materials can also be synthesized by self-assembly of positively charged surfactant micelles. The dodecagonal quasicrystal structure is the last in a sequence of four different structures discovered in this study as a function of either micelle pore expander concentration or reaction stirring rate. Via direct visualization employing cryo-transmission electron microscopy (cryo-TEM) and quantitative image analysis, we find that quasicrystallinity is predominantly governed by asymmetries in the micelle size distribution and identify micelle pore expander concentration and reaction stirring rate as equivalent experimental control parameters. Furthermore, precise control of the silica chemistry enables MSN sizes to be tuned from above $100 \mathrm{~nm}$, where dodecagonal tiling patterns are well developed, to below $30 \mathrm{~nm}$, where particles consisting of only a single triangle or square tiling unit represent the earliest formation stages of the dodecagonal tiling. Experimental results are then compared to growth simulations. Our results suggest that the incorporation of building blocks with asymmetric size distributions at early times in the growth process promotes quasicrystal formation and that simple synthesis parameters like stirring rate can be used for their control.

\section{Results}

[TMB]-induced transitions between four MSN structures. MSNs were synthesized by co-condensing a mixture of silane precursors, tetraethyl orthosilicate (TEOS) and N-(2-aminoethyl)-3-aminopropyltrimethoxysilane (AEAPTMS), in the presence of hexadecyltrimethylammonium bromide (CTAB) surfactant micelles (see molecular structures in Fig. 1a). The aminosilane was added to tailor the packing behavior of the CTAB surfactant molecules 24,25 , enabling access to more complex silica nanostructures $19,20,26$. The CTAB micelle size and size distribution was systematically varied by adjusting the concentration of a micelle pore expander, mesitylene (TMB ${ }^{27-29}$. TEM images show that as the concentration of TMB, [TMB], was increased, the MSN structure changed from hexagonal (Fig. 1b) to multicompartment (Fig. 1c and Supplementary Fig. 1) to cubic (Fig. 1d) and finally to a structure with dodecagonal symmetry (Fig. 1e).

Small-angle X-ray scattering (SAXS) measurements, which average over macroscopic volumes, were performed on the TEOS/AEAPTMS particles (Supplementary Fig. 2). The results corroborate the structural transitions observed by TEM (Fig. 1b-e). At $12 \mathrm{mM}$ [TMB], the SAXS pattern shows reflections consistent with hexagonal symmetry. When [TMB] is increased to $29 \mathrm{mM}$, a well-resolved set of cubic $P m \overline{3} n$ reflections from the particle cores is superposed with a prominent set of hexagonal $p 6 \mathrm{~mm}$ reflections from the particle arms. This pattern is consistent with previously reported SAXS measurements from multicompartment MSNs ${ }^{19}$. At $47 \mathrm{mM}$ [TMB], the SAXS pattern shows reflections consistent with a cubic particle with $\operatorname{Pm} \overline{3} n$ lattice ${ }^{24}$. Expected peak positions for cubic $P m \overline{3} n$ and hexagonal $p 6 \mathrm{~mm}$ are indicated in the SAXS patterns using solid and dashed lines, respectively (Supplementary Fig. 2). As [TMB] is further increased to $116 \mathrm{mM}$ and beyond, the SAXS patterns lose more and more features and lattices cannot unambiguously be assigned. TEM tilt series (Supplementary Fig. 3) as well as TEM tomography failed to establish the three-dimensional (3D) particle structure, the latter as a result of severe beam damage of the organic-silica hybrids during data acquisition.

To deconvolute the effects of [TMB] from the specifics of the silica chemistry, we examined a second, independent organosilane system, composed of tetramethyl orthosilicate (TMOS) and (3-aminopropyl)trimethoxysilane (APTMS) (Fig. 1a). MSNs synthesized using TMOS and APTMS showed similar transitions as a function of increasing [TMB] (Fig. 1f-i). Comparison between the TEOS/AEAPTMS and TMOS/APTMS systems suggests that the observed structural transitions do not depend on the specifics of the silane and aminosilane molecules used here. However, it is important to note that the presence of an aminosilane is critical for the formation of quasicrystalline MSNs (Supplementary Fig. 4a, b). This observation is consistent with our expectation that the aminosilane mediates changes in the packing behavior of the CTAB surfactant ${ }^{24,25}$, facilitating the formation of more complex nanostructures $19,20,26$.

Pore patterns of MSNs like the ones in Fig. 1e were tiled using squares and equilateral triangles (Fig. 2a, b). These MSNs exhibit two distinctive features of dodecagonal quasicrystals. Fast Fourier transform (FFT) analysis of TEM images reveals 12-fold symmetry and no translational periodicity is observed in the two-dimensional (2D) tiling patterns extracted from the TEM images (Fig. 2a, b) ${ }^{11}$. The tile edges correspond to dodecagonal directions and each vertex can be described by $\mathbf{r}^{/ /}=n_{1} \mathbf{e}_{1}^{/ /}+$ $n_{2} \mathbf{e}_{2}^{/ /}+n_{3} \mathbf{e}_{3}^{/ /}+n_{4} \mathbf{e}_{4}^{/ /}$(Fig. 2b). Each vertex in parallel (Fig. 2c) space corresponds to a unique vertex in perpendicular (Fig. 2d) space $^{30}$. Figure $2 \mathrm{c}$ and $\mathrm{d}$ show by color how individual points move during this transformation. A comparison of the radii of gyration in perpendicular and parallel space, $R_{g} \perp$ vs. $R_{g} / /$, is a 
a
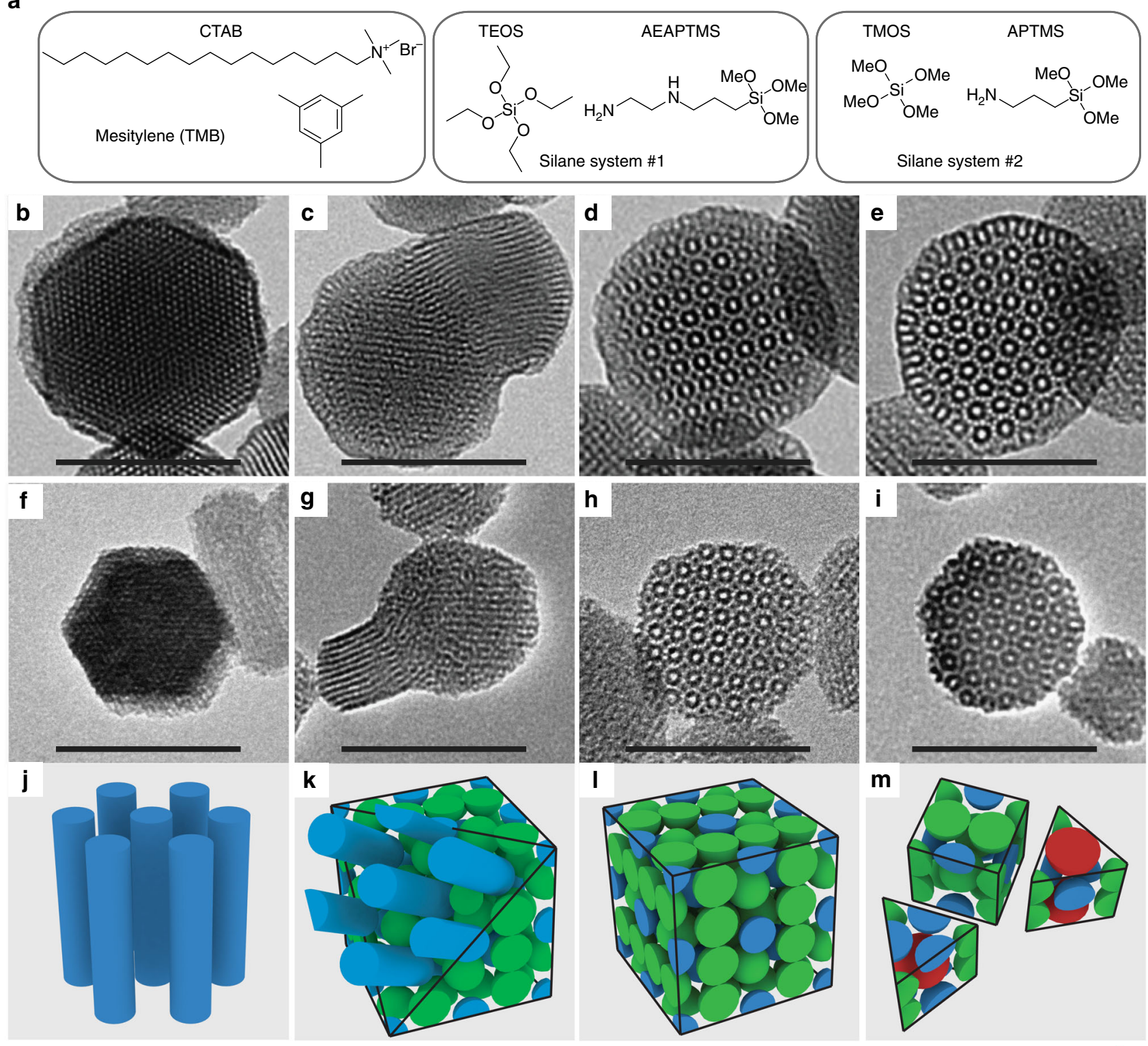

Increasing $[\mathrm{TMB}]$

Fig. 1 Four MSN structures observed in two silane systems as a function of mesitylene concentration. a Chemical structures of key reagents used in TEOS/AEAPTMS and TMOS/APTMS synthesis systems. b-e TEM images of MSNs prepared from TEOS/AEAPTMS with $12 \mathrm{mM}$ (b), $29 \mathrm{mM}$ (c), $47 \mathrm{mM}$ (d), and $116 \mathrm{mM}$ (e) mesitylene (TMB), using constant ammonium hydroxide concentration (13.8 mM) and stirring rate (650 rpm). $\mathbf{f - i}$ TEM images of MSNs prepared from TMOS/APTMS with $4 \mathrm{mM}(\mathbf{f}), 10 \mathrm{mM}(\mathbf{g}), 14 \mathrm{mM}(\mathbf{h})$, and $72 \mathrm{mM}$ (i) TMB, using constant ammonium hydroxide concentration $(150 \mathrm{mM})$ and stirring rate $(600 \mathrm{rpm})$. $\mathbf{j}-\mathbf{m}$ Micelle packing models corresponding to MSNs in $\mathbf{b}$-i. All scale bars are $100 \mathrm{~nm}$

measure of the quasicrystallinity of a pattern: the more a pattern shrinks, the more quasicrystalline it is (compare patterns in Fig. 2c-d).

Synthesis of MSNs with varying size and quasicrystallinity. We systematically investigated the growth of dodecagonal tilings in mesoporous silica all the way down to a single tiling unit by capitalizing on well-established silica chemistry to control the particle size. The comparatively fast hydrolysis of TMOS relative to TEOS facilitated access to smaller MSNs ${ }^{21}$. Precise particle size control in this system was further achieved using variations in reaction $\mathrm{pH}$, where the increasing condensation rate associated with lowered $\mathrm{pH}$ resulted in smaller particle sizes ${ }^{31}$. In this way, MSNs could be synthesized with sizes $<30 \mathrm{~nm}$ such that associated tilings were composed of a single triangle or square tiling unit. TEM images of quasicrystalline MSNs, synthesized using constant [TMB] and varying ammonium hydroxide concentration, $\left[\mathrm{NH}_{4} \mathrm{OH}\right]$, are shown in the bottom row of Fig. 2e. Interestingly, qualitative assessment of the triangle-square tilings extracted from these images suggests that quasicrystallinity does not vary with changes in reaction $\mathrm{pH}$.

Increasing [TMB] from 14 to $72 \mathrm{mM}$ introduces a gradual transition from cubic to quasicrystalline pore structure, where triangle-square tilings extracted from MSNs synthesized using intermediate $[\mathrm{TMB}]$ are mixed phase rather than purely cubic or 

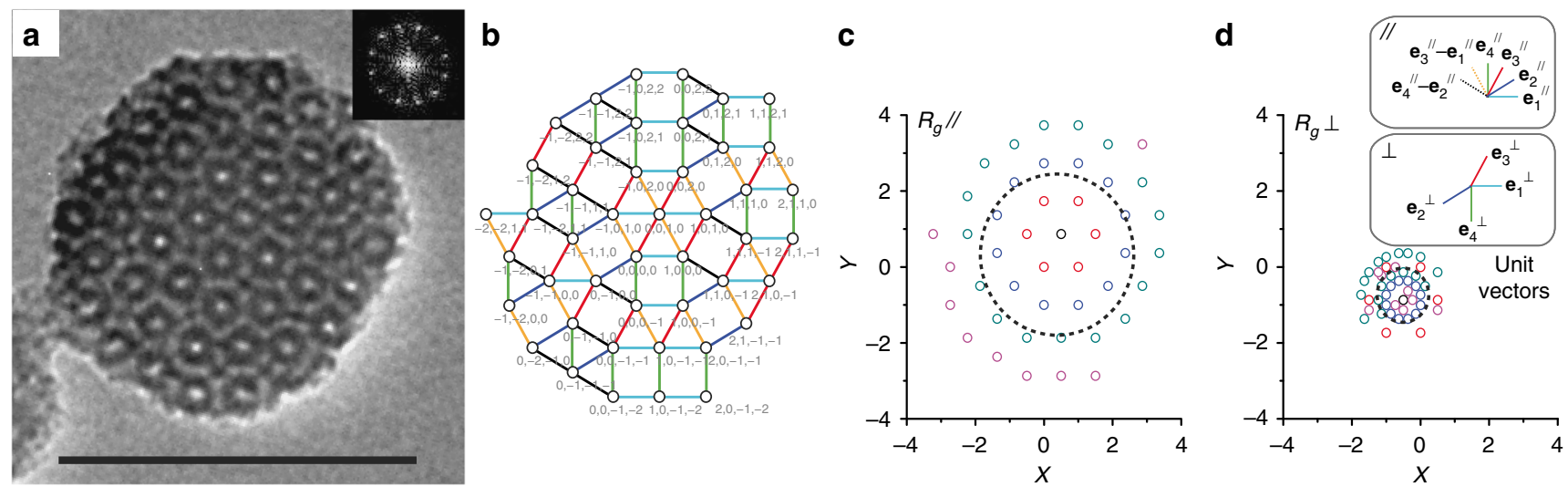

e

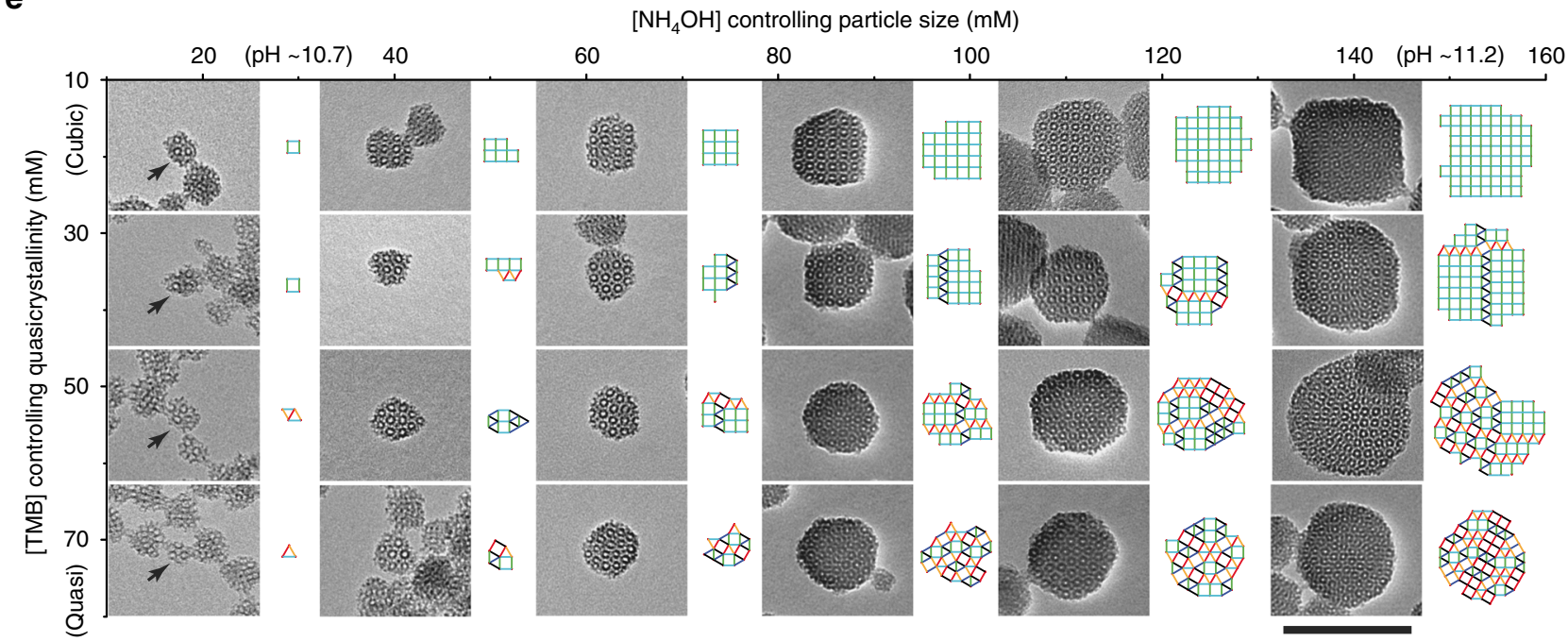

Fig. 2 Quasicrystallinity of TMOS/APTMS-derived MSNs. a TEM image of a MSN with dodecagonal symmetry, synthesized using TMOS and APTMS. The scale bar is $100 \mathrm{~nm}$. The insert in a shows a diffractogram of the TEM image exhibiting 12-fold symmetry. b Square-triangle tiling and parallel space coordinates of the nanoparticle shown in a. Edges with different colors correspond to the six dodecagonal directions in parallel space. The corresponding dodecagonal directions in parallel and perpendicular space are shown in d. c, d Parallel and perpendicular space coordinates for the triangle-square tiling shown in $\mathbf{b}$. Dotted circles with radius equal to $R_{g} / /$ and $R_{g} \perp$ are shown on the parallel and perpendicular space plots, respectively. The colors in $\mathbf{c}$ and d show how individual points move during the transformation. e TEM images and corresponding square-triangle tilings of representative MSNs (arrows) synthesized from different concentrations of mesitylene $(T M B)$ and ammonium hydroxide $\left(\mathrm{NH}_{4} \mathrm{OH}\right)$. The stirring rate was 600 rpm for all syntheses. All images have the same magnification; scale bar is $100 \mathrm{~nm}$

quasicrystalline. Tuning reaction $\mathrm{pH}$ at low and intermediate [TMB] again resulted in particle sizes ranging from $>100 \mathrm{~nm}$ down to $<30 \mathrm{~nm}$ with no observable $\mathrm{pH}$-dependent variations in pore structure. Figure 2e illustrates the range of particle structures obtained from over 50 synthesis batches of particles (see also Supplementary Fig. 4).

Quantitative analysis of micelle size distributions. Although the observed MSN structures result from cooperative interactions between the CTAB micelles and the silica species $^{32}$, the above observations point to the starting micelle size distribution as a key factor in the formation of quasicrystalline MSNs. Recent reports provide supporting evidence for the role of building block polydispersity in self-assembly, demonstrating that particles with broad monomodal or bimodal size distributions form more complex structures $7,8,33$. We have characterized the starting micelle sizes using cryo-TEM (Fig. 3). Representative micelle size distributions from native $\mathrm{CTAB} / \mathrm{TMB}$ solutions prepared using increasing $[\mathrm{TMB}]$ and constant stirring rate are shown in
Fig. 3a-d. Micelle solutions prepared without TMB or using low [TMB] (4 mM) led to formation of hexagonal MSNs (Fig. 3k, l), whereas use of high [TMB] (72 and $116 \mathrm{mM})$ resulted in quasicrystalline MSNs (Fig. $3 \mathrm{~m}, \mathrm{n}$ ). Comparison of the entire series reveals that increasing [TMB] causes a shift to larger average micelle diameter as well as an increase in the positive skewness of the micelle size distribution, e.g., compare numbers of micelles with sizes above $5 \mathrm{~nm}$ as [TMB] increases (Fig. 3a-d).

Cryo-TEM experiments further demonstrated that mechanical agitation (stirring), in addition to TMB, is a requirement for producing these broadened micelle size distributions (Fig. 3e, j, o), consistent with recent observations of complex polymer micelle size distributions in the presence of a co-solvent and solution agitation $^{34}$. Associated structural transitions are illustrated schematically by the micelle packings in Fig. $1 \mathrm{j}-\mathrm{m}^{6,35,36}$, using a model with three discrete micelle sizes for simplicity. Hexagonal MSNs have channel-like pores templated by elongated CTAB micelles (blue) (Fig. 1j). Multicompartment MSNs are composed of a $\operatorname{Pm} \overline{3} n$ cubic core connected epitaxially at the (111) face to a hexagonal structure ${ }^{19}$. The $P m \overline{3} n$ cubic core is composed of two 
types of spherical micelles, one of which is slightly larger (green) than the other (blue) due to increased TMB loading (Fig. 1k). As [TMB] is further increased, subsequently single phase $P m \overline{3} n$ cubic MSNs (Fig. 11) and MSNs with dodecagonal symmetry are formed, the latter incorporating even larger micelles (red) (Fig. $1 \mathrm{~m}$ ).

These observations are supported by pore size analysis of nitrogen sorption measurements on TEOS/AEAPTMS particles after $\mathrm{CTAB}$ removal that show marked pore size increase and broadening of the pore size distribution with increasing [TMB] (Supplementary Fig. 2). At $12 \mathrm{mM}$ (hexagonal MSNs), the pore size distribution is well fit by a single log-normal distribution (Supplementary Fig. 5). However, as [TMB] is increased to 47 and $116 \mathrm{mM}$ (cubic and quasicrystalline MSNs), the increasingly broadened pore size distributions can no longer be described by unimodal log-normal fits, suggesting that additional micelle populations may be involved in the formation processes of these more complex structures. As a first approximation, these data are therefore fit using a superposition of two and three log-normal distributions, respectively (Supplementary Fig. 5). Average pore sizes extracted from these fits (Supplementary Table 1) support the hypothesis that the structural changes observed with increasing [TMB] are due to the presence of additional, larger micelle populations. Additional experiments demonstrated that the same structural transitions and pore size increases were achieved using constant [TMB] $(29 \mathrm{mM})$ but increased stirring rates that also affects micelle size (Supplementary Figs. 5, 6).

Tiling analysis. The MSNs shown in Fig. 1e, i exhibit indicators of quasicrystallinity (i.e., 12-fold symmetric diffractogram and no translational periodicity). Since there are no local constraint rules that enforce quasicrystallinity in systems of triangles and squares, quasicrystallinity in these finite structures is instead characterized by statistical analysis of the phason coordinates. We analyzed the tilings extracted from the TMOS/APTMS-derived MSNs synthesized as a function of increasing [TMB] (Fig. 2e and Supplementary Fig. 5). Figure 4a shows a plot of $R_{g} \perp$ vs. $R_{g} / /$ calculated from the tilings of individual MSNs synthesized with a
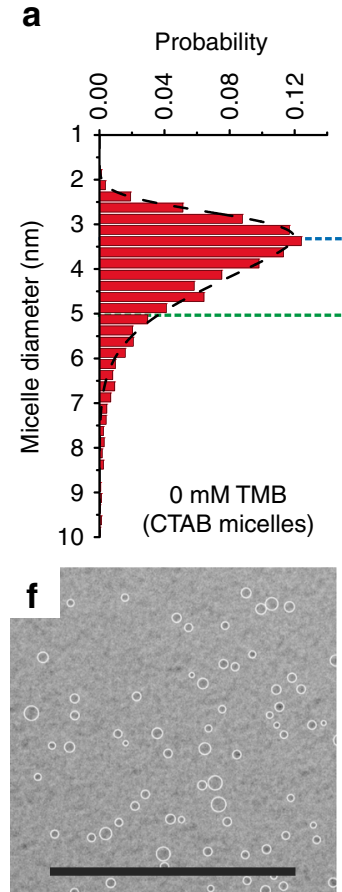

k

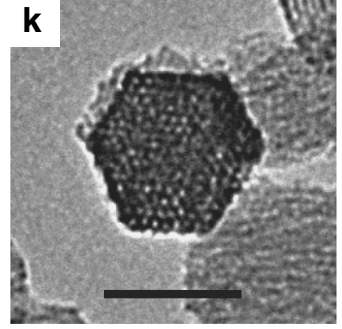

b
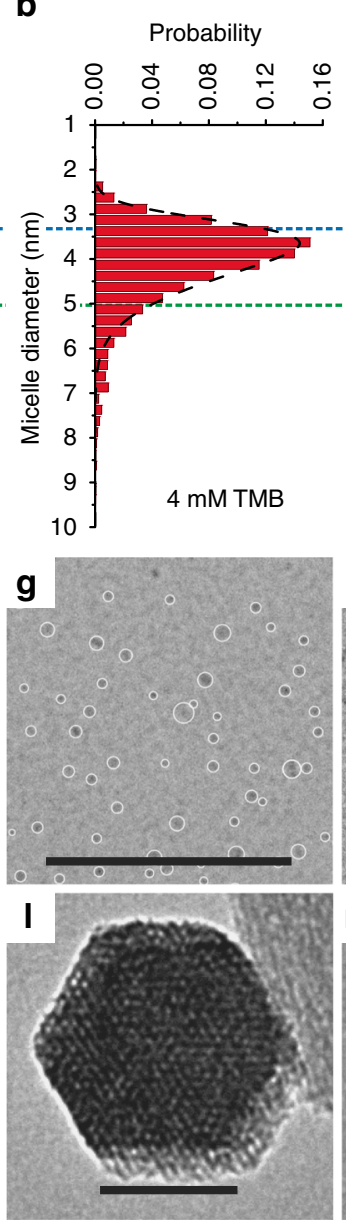

C
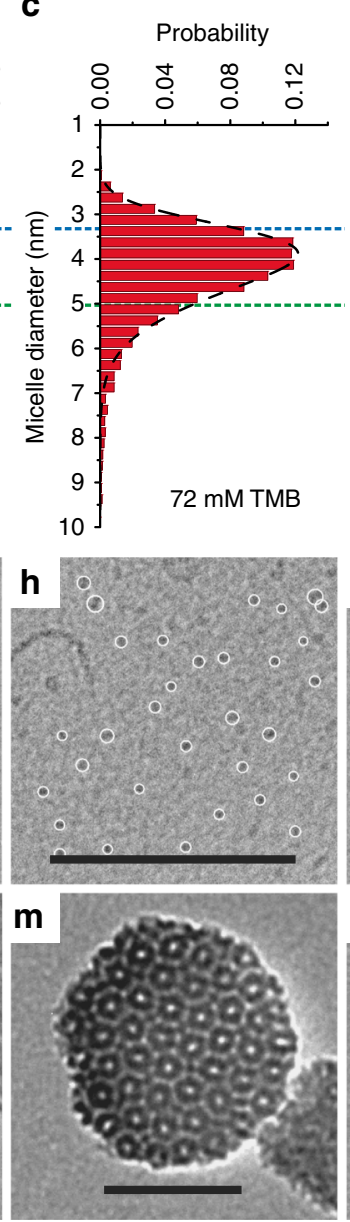

d
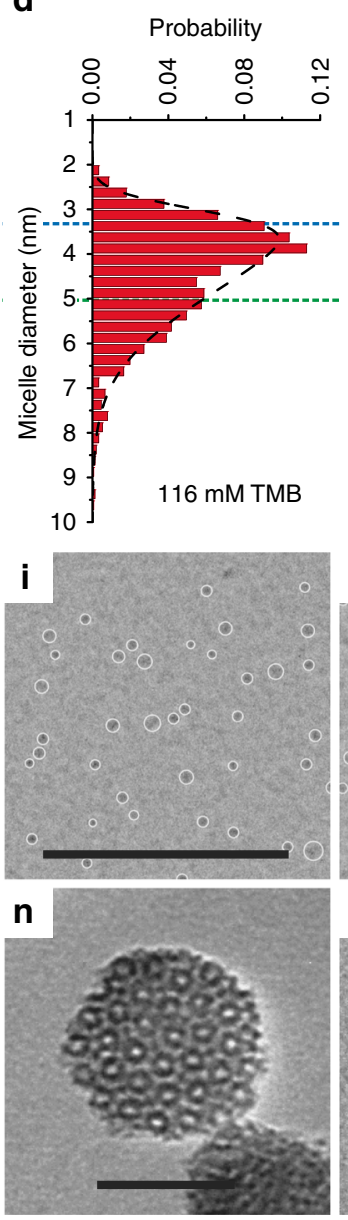

e
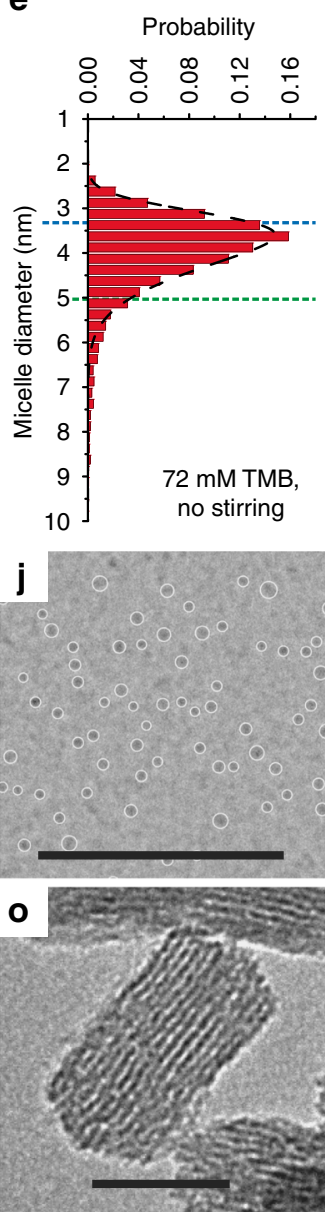

Fig. 3 Cryo-TEM of native micelle solutions prepared using varying mesitylene concentrations and stirring rates. a-e Micelle size distributions before silane

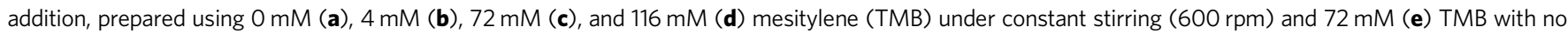
stirring. The ammonium hydroxide concentration was kept constant ( $150 \mathrm{mM}$ ) across all solutions. Distribution fits are shown using black dashed lines. Blue dashed lines indicate the peak position of the micelle size distribution of CTAB micelles without TMB and green dashed lines are shown as an additional reference for comparison across different plots. Comparison between the micelle size distributions demonstrates that although the peak position does not vary

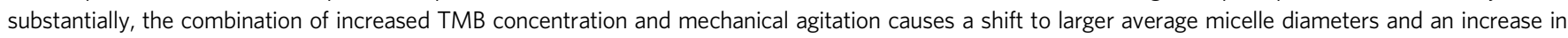
the frequency of large micelles with sizes above $5 \mathrm{~nm}$. $\mathbf{f}-\mathbf{j}$ Cryo-TEM images of the micelle solutions before silane addition corresponding to conditions used in

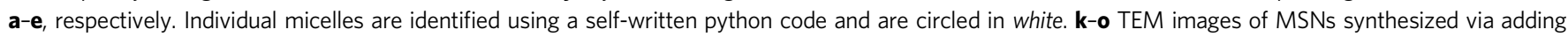
TMOS and APTMS into micelle solutions corresponding to conditions used in a-e, respectively. Scale bars in both sets of images are 50 nm 
systematic variations in $[\mathrm{TMB}]$ and $\left[\mathrm{NH}_{4} \mathrm{OH}\right]$. The slope of the linear fit $R_{g} \perp=A R_{g} / /+B$ provides the magnitude of the phason strain, a quantitative measure of quasicrystallinity ${ }^{6,30}$. MSNs synthesized at the highest [TMB] $(72 \mathrm{mM})$ and varying $\mathrm{pH}$ lie on the same line, which is well described by the fit with phason strain equal to 0.14 (see TEOS/AEAPTMS data in Supplementary Fig. 7) approaching the zero-phason strain of a dodecagonal quasicrystal $^{6}$ and consistent with the phason behavior of a random tiling quasicrystal ${ }^{30}$. Additionally, the fit demonstrates that these MSNs have similar quasicrystallinity, which contrary to previous studies ${ }^{6}$, is independent of the reaction $\mathrm{pH}$ and $\mathrm{pH}$ induced changes in the interaction potentials between micelles.

TEM images of MSNs synthesized using low [TMB] and varying $\mathrm{pH}$ (Fig. 2e and Supplementary Fig. 4) exhibit predominantly cubic structure. Interestingly, a few quasicrystalline MSNs are always found in a batch of cubic MSNs, resulting in a slope that is slightly smaller than 1, i.e., the phason strain of a perfectly cubic crystal. Comparison between MSNs synthesized using low and high [TMB] demonstrates that changes in quasicrystallinity are due to differences in [TMB] rather than $\mathrm{pH}$. Particles synthesized at intermediate [TMB] between 22 and $50 \mathrm{mM}$ exhibited substantial fluctuations in the plot of $R_{g} \perp$ vs. $R_{g} / /$ (Fig. 4a). More specifically, individual particles from a single batch of MSNs synthesized in this regime may be cubic, quasicrystalline, or mixed phase, suggesting that not only [TMB] and stirring rate but also other factors are at play.

From the tiling analysis the distribution of four fundamental geometrical conformations was derived for all batches/particles, i.e., $4^{4}, 3^{3} 4^{2}, 3^{2} 4^{1} 3^{1} 4^{1}$, and $3^{6}$, where 4 and 3 represent square and triangle base units and the exponent indicates the number of adjacent base units incident on a single vertex (Fig. 4b). This analysis shows that with increasing [TMB], cubic $4^{4}$ conformers decrease, $3^{3} 4^{2}$ conformers first increase but then roughly stay constant, and $3^{2} 4^{1} 3^{1} 4^{1}$ conformers increase. All MSN samples analyzed have low abundance of $3^{6}$ conformers. At the same time, the ratio of the overall numbers of triangles to squares increases with [TMB] (Fig. 4c). Considering that the triangle tile contains the largest micelles (Fig. 1m), these data imply that the presence of large micelles plays a key role in quasicrystal formation. The growth of quasicrystals may also be highly dependent on the dynamic packing process of differently sized micelles forming triangle and cubic tiles, as indicated by the substantial phason strain fluctuations of the MSNs synthesized at intermediate [TMB]. Simulations were carried out in order to elucidate the origin of these fluctuations and reveal dominant factors in this [TMB] regime.

Simulated single-particle growth trajectories. Quasicrystal growth has previously been modeled in two dimensions using triangle-square tilings ${ }^{6}, 13,37$. We have developed an irreversible MSN growth model that produces 2D tilings that mimic the observed features of our experimental system (see Supplementary Methods for details). Particle growth in these simulations proceeds via irreversible aggregation of square and equilateral triangle tiles. This accounts for experimental observations wherein packings of small and medium-sized micelles result in square tiles (green and blue in Fig. 11) and the involvement of a third, larger micelle gives rise to triangle tiles ( red in Fig. $1 \mathrm{~m}$ ). Each tile is randomly chosen from a weighted shape distribution given by an input triangle/square number ratio that reflects the increasing number of triangles observed in MSNs synthesized at increasing [TMB] (Fig. 4b, c). The probability of tile attachment on the growing cluster is governed by both shape and site-specific interaction parameters. We also include an additional threshold parameter that allows the system to choose replacement tiles if attachment probabilities above a minimum value do not exist. For each triangle/square ratio, several batches of particles with size distributions that approximately match those of experimentally observed particles were generated. Simulated particles were analyzed according to the methods outlined for the experimental particles. Although this is a simplified model, comparison of Fig. 4d through $\mathrm{f}$ with Fig. 4a through $\mathrm{c}$ shows that it was sufficient to reproduce the experimentally observed features. These a

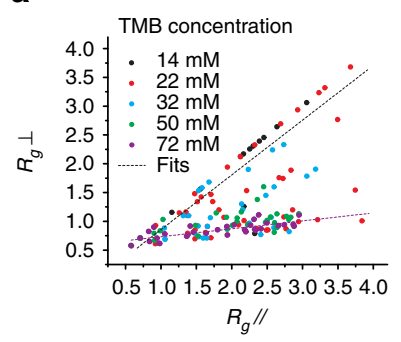

d

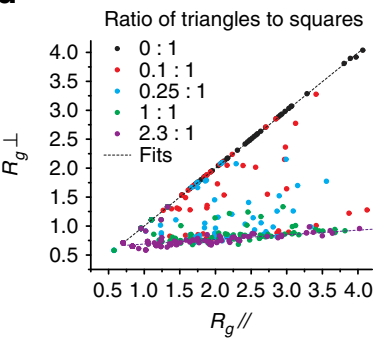

b

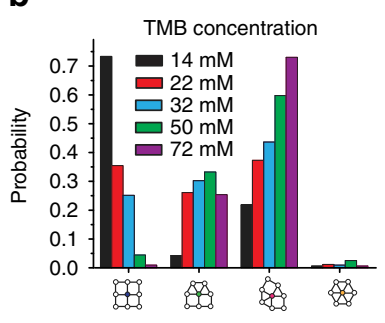

e

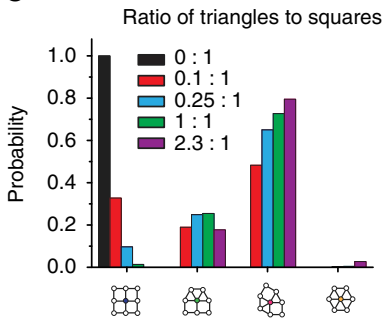

C
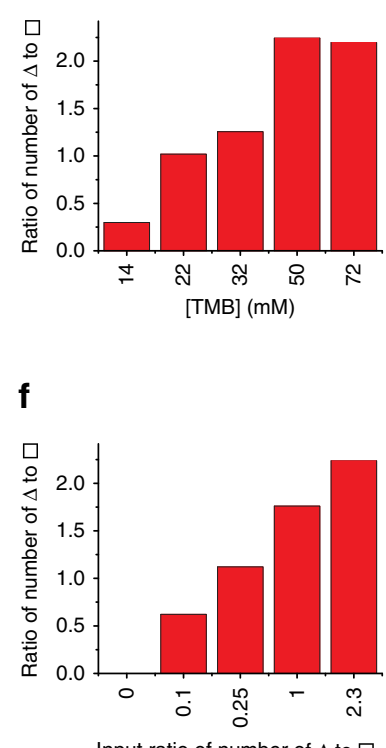

Fig. 4 Analysis of TMOS/APTMS-derived MSNs and simulated structures. a Plot of $R_{g}$ in perpendicular vs. parallel space, calculated for MSNs synthesized from five different mesitylene (TMB) concentrations. b Distribution of pore conformers $\left(4^{4}, 3^{3} 4^{2}, 3^{2} 4^{1} 3^{1} 4^{1}\right.$, and $\left.3^{6}\right)$ calculated for particles synthesized from five different TMB concentrations. c Ratio of triangle to square tiles calculated for particles in $\mathbf{a}$. $\mathbf{d}-\mathbf{f}$ Plots corresponding to $\mathbf{a}-\mathbf{c}$ for 500 particles derived from simulations with five different triangle to square ratios 
results suggest that the structural transition observed is primarily a function of the triangle/square ratio or micelle size distribution in an irreversible, random growth process.

Analysis of simulated single-particle growth trajectories (Fig. 5a) shows the relationship between quasicrystallinity $\left(R_{g} \perp\right.$ vs. $\left.R_{g} / /\right)$ and the size of the particle or the number of square tiles present (plotted along the $z$-axis) when the first triangle is added. For perfect cubic particles, the total number of squares at the end of the simulation is counted instead. At the highest triangle/square input ratio of $2.3^{38}$, all data points (purple) are highly concentrated in a low $z$-range in Fig. 5a, suggesting that in this irreversible tiling process the early appearance of a first triangle contributes to the growth of highly quasicrystalline particles. By comparison, the appearance of the first triangle in particles with a triangle/square input ratio of $0.1(\mathrm{red})$ is widely distributed in the growth process causing the spread in quasicrystallinity observed in Fig. 4d. 2D projections of Fig. 5a, which represent the dependence of $R_{g} / /$ and $R_{g} \perp$ on the timing (i.e., particle size) of the first triangle addition, are shown in Fig. 5b and c, respectively. In Fig. 5c, all data points fall onto a master curve, $N=N\left(R_{g} \perp\right)$, that relates the area of a $2 \mathrm{D}$ square tiling pattern $(\propto N)$ to its radius of gyration (see Supplementary Methods).

Side-by-side comparison of Fig. $5 b$ and $c$ reveals that the first triangle addition seeds the growth of a quasicrystalline phase. While particle growth in real (parallel) space does not depend on the timing of the triangle addition, particle growth in perpendicular space is mostly terminated as soon as the first triangle is added. Single-particle growth trajectories (Fig. 6) of two simulated particles with the same triangle/square input ratio (0.1) illustrate this effect. Similarities between tilings from TEM images of particles synthesized at intermediate [TMB] and tilings of simulated particles at various stages of growth support the assumption that the formation of MSNs with dodecagonal tilings can be described using our model (Fig. 6).

\section{Discussion}

We have discovered a series of four different structures in MSNs, including dodecagonal tiling, as a function of either pore expander concentration or stirring rate. Precise control of highly tunable silica sol-gel chemistry together with growth simulations has enabled identification of the early growth trajectories of quasicrystalline MSNs. Our results suggest that skewed micelle size distributions as mapped by cryo- TEM frustrate crystal formation and favor the growth of quasicrystalline structures. Our experiments further suggest that this skewness can be induced either by increasing amounts of micelle pore expander, or by simple increase of stirring rate. These results imply that size polydispersity of building blocks, rather than $\mathrm{pH}$-dependent changes in interaction potentials between building blocks, as suggested earlier ${ }^{6}$, is the primary factor in triggering the growth of quasicrystalline mesoporous silica structures.

Our results suggest that due to fast silane condensation, quasicrystalline MSNs are likely formed via an irreversible micelle packing process. Although the micelles studied here are unlikely to undergo the local structural rearrangements that are observed in metal alloys during high temperature processing steps ${ }^{14}$, we expect that the simple synthetic control parameters we have identified can be translated to a wide variety of selfassembled systems. The accessibility of the experimental control parameters we introduce in this study may simplify the design of alternative self-assembly chemistries for studying quasicrystal growth, especially in systems of polymer micelles where addition of co-solvents and mechanical agitation have already been shown to affect building block polydispersity ${ }^{34}$. Finally, although we are limited by the silica chemistry to relatively small particle sizes, the experimental observation of quasicrystal formation via an irreversible growth mechanism may provide fundamental insights into the origin of quasicrystallinity in other materials systems.

\section{Methods}

Materials. CTAB ( $\geq 99 \%)$, ethyl acetate (EtOAc, ACS grade), ammonium hydroxide $\left(\mathrm{NH}_{4} \mathrm{OH}, 29 \%\right)$, 1,3,5-trimethylbenzene (TMB, 99\%), AEAPTMS (95\%), APTMS (95\%), TEOS ( $\geq 99 \%)$, TMOS ( $\geq 99 \%)$, hydrochloric acid ( $\mathrm{HCl}, 36.5-38 \%)$, acetic acid (glacial), and ethanol (absolute, anhydrous) were used as received without further purification. Deionized water (Milli-Q, $18.2 \mathrm{M} \Omega \mathrm{cm}$ ) was used throughout.

Synthesis of TEOS/AEAPTMS-derived MSNs. TEOS/AEAPTMS-derived MSNs with different structures were synthesized in aqueous solution at room temperature through surfactant-directed silica condensation. Round bottom flasks and egg-shaped stir bars were used in all TEOS/AEAPTMS syntheses. MSNs were prepared with [TMB] ranging from 12 to $205 \mathrm{mM}$. EtOAc $(0.44 \mathrm{~mL}), \mathrm{NH}_{4} \mathrm{OH}$ $(1.35 \mathrm{~mL})$, and then TMB were added to an aqueous solution of CTAB $(52.5 \mathrm{~mL}$, $2.61 \mathrm{mM})$ stirred at $650 \mathrm{rpm}$. After $30 \mathrm{~min}$, a mixture of TEOS $(0.25 \mathrm{~mL})$ and AEAPTMS $(0.0375 \mathrm{~mL})$ was added to the reaction under continued stirring and allowed to react for $5 \mathrm{~min}$. Then, additional water $(18.45 \mathrm{~mL})$ was added and the reaction was left stirring for $24 \mathrm{~h}$. On completion of the reaction, the particle suspension was neutralized with $2 \mathrm{M} \mathrm{HCl}$, before cleaning by repeated centrifugation and redispersion in EtOH. CTAB was removed from the resulting particles by adding $5 \mathrm{vol} \%$ of acetic acid to the suspension and stirring for $30 \mathrm{~min}$. Following CTAB removal, the particles were again cleaned by repeated centrifugation and redispersion in $\mathrm{EtOH}$.

In order to study the effect of stirring rate on the structure of these particles, MSNs were synthesized using constant [TMB] $(29 \mathrm{mM})$ and four different stirring rates: $500 \mathrm{rpm}, 650 \mathrm{rpm}, 800 \mathrm{rpm}$, and $1000 \mathrm{rpm}$. All other synthesis parameters were unchanged from the above description.

Synthesis of TMOS/APTMS-derived MSNs. TMOS/APTMS-derived MSNs of different sizes and structures were synthesized by surfactant-directed silica
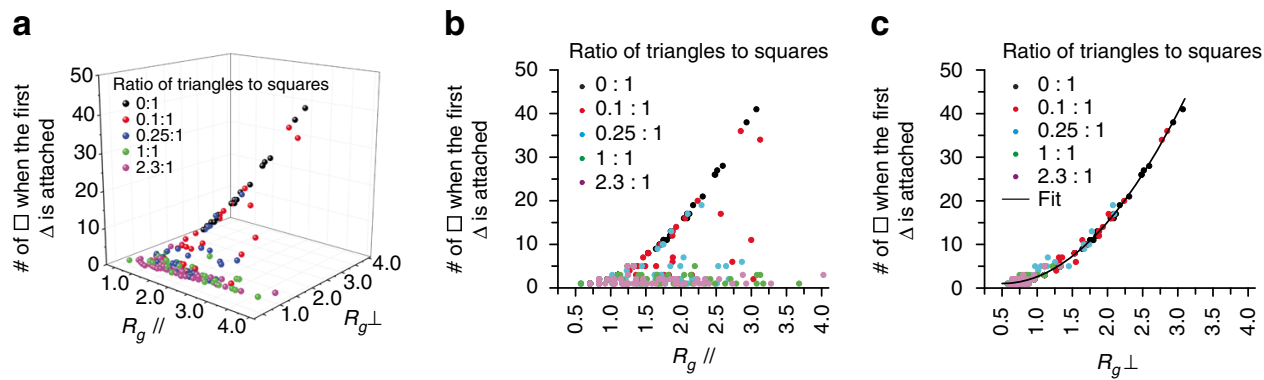

Fig. 5 Analysis of simulated single-particle growth trajectories. a 3D plot showing that phason strain is highly dependent on the time at which the first triangle is added to the growing particle. $\mathbf{b}, \mathbf{c} 2 \mathrm{D}$ projections of the $3 \mathrm{D}$ plot in $\mathbf{a}$. The data in $\mathbf{c}$ is fitted using equation $N a^{2}=A R_{g} \perp^{2}+a B R_{g} \perp+a^{2} C$, where $a=$ 1 is the edge length of a square tile, $N$ is the number of tiles, and $A, B$, and $C$ are fitting parameters. This is consistent with the theoretical relationship between the area and the radius of gyration, $R_{g}$, of a $2 \mathrm{D}$ square packing pattern (Supplementary Methods) 
condensation in room temperature, aqueous solution. Round bottom flasks and egg-shaped stir bars were used in all TMOS/APTMS syntheses. MSNs were prepared with [TMB] ranging from 4 to $116 \mathrm{mM}$ and $\left[\mathrm{NH}_{4} \mathrm{OH}\right]$ ranging from 15 to $150 \mathrm{mM}$. High [TMB] resulted in more quasicrystalline particles and high $\left[\mathrm{NH}_{4} \mathrm{OH}\right]$ resulted in larger particle sizes. $\mathrm{NH}_{4} \mathrm{OH}$ and TMB were added to an aqueous solution of CTAB $(10 \mathrm{~mL}, 22.78 \mathrm{mM})$ stirred at $600 \mathrm{rpm}$. After $2 \mathrm{~h}$, a mixture of TMOS $(34 \mu \mathrm{L})$ and APTMS $(25 \mu \mathrm{L})$ was added to the reaction and allowed to react for $24 \mathrm{~h}$ under continued stirring. Applied synthesis conditions gave access to particle sizes between about 30 and $150 \mathrm{~nm}$. On completion of the reaction, the particle suspension was cleaned by repeated centrifugation and redispersion in EtOH. CTAB was removed from the resulting particles by adding 5 vol\% of acetic acid to the suspension and stirring for $30 \mathrm{~min}$. Following CTAB removal, the particles were again cleaned by repeated centrifugation and redispersion in EtOH. For the APTMS study (Supplementary Fig. 4), the $\left[\mathrm{NH}_{4} \mathrm{OH}\right]$ was held constant at $120 \mathrm{mM}$ and the APTMS concentration was varied from 0 to $14 \mathrm{mM}$. All other parameters were kept the same.

Characterization. TEM images were taken using a FEI Tecnai T12 Spirit microscope operated at an acceleration voltage of $120 \mathrm{kV}$. FFT analysis was performed using ImageJ software. Each TEM sample was prepared after CTAB removal by evaporating $10 \mu \mathrm{L}$ of suspension on TEM grid in dry air. Cryo-TEM images were collected under low-dose conditions using a customized FEI Titan

a
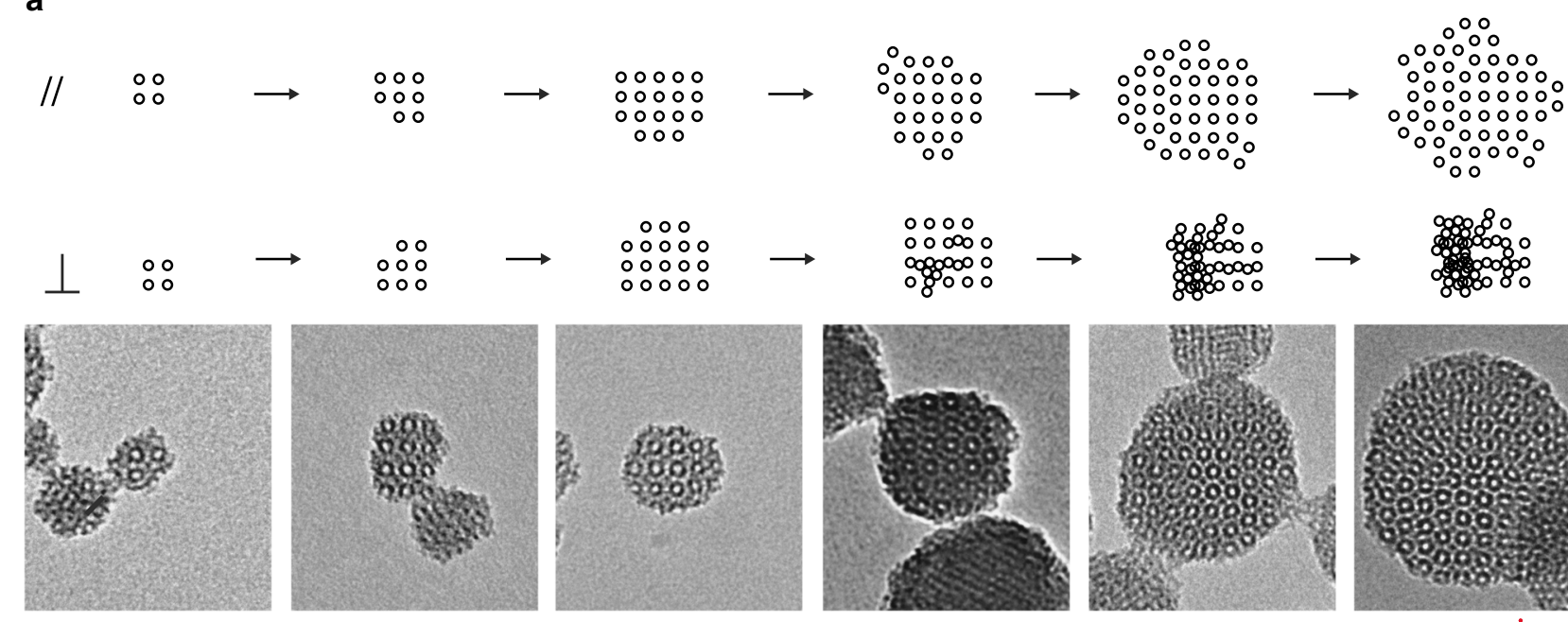

$0000 \%$

00
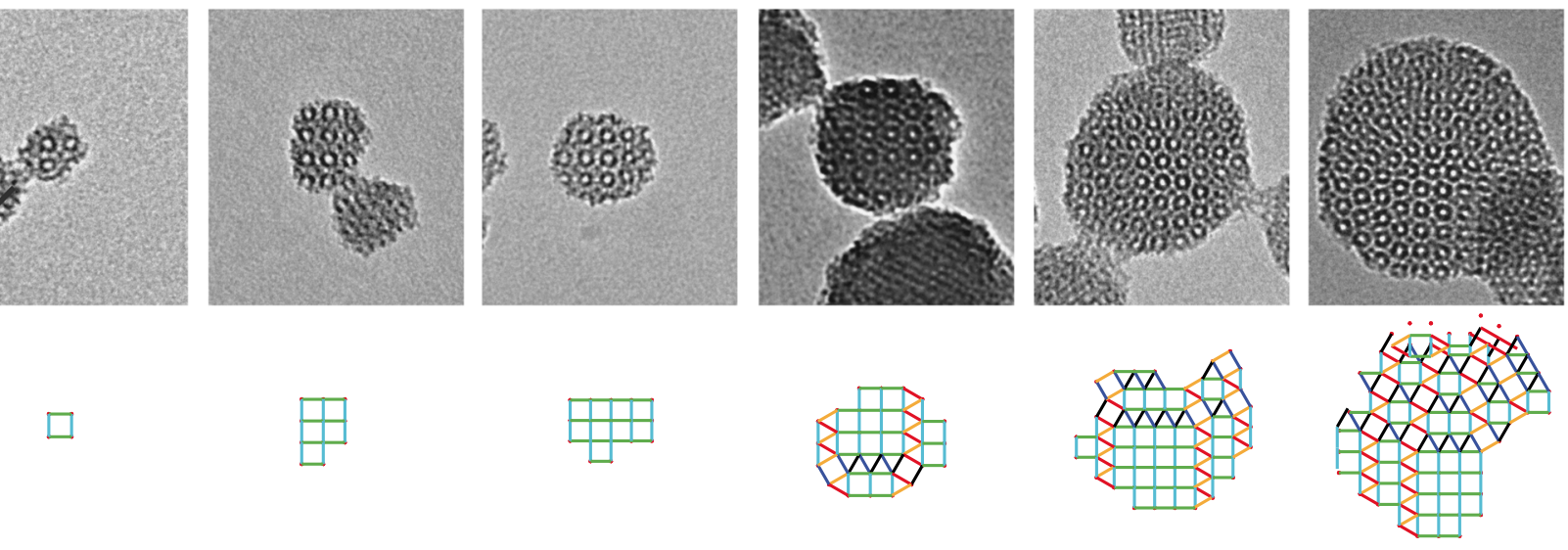

b

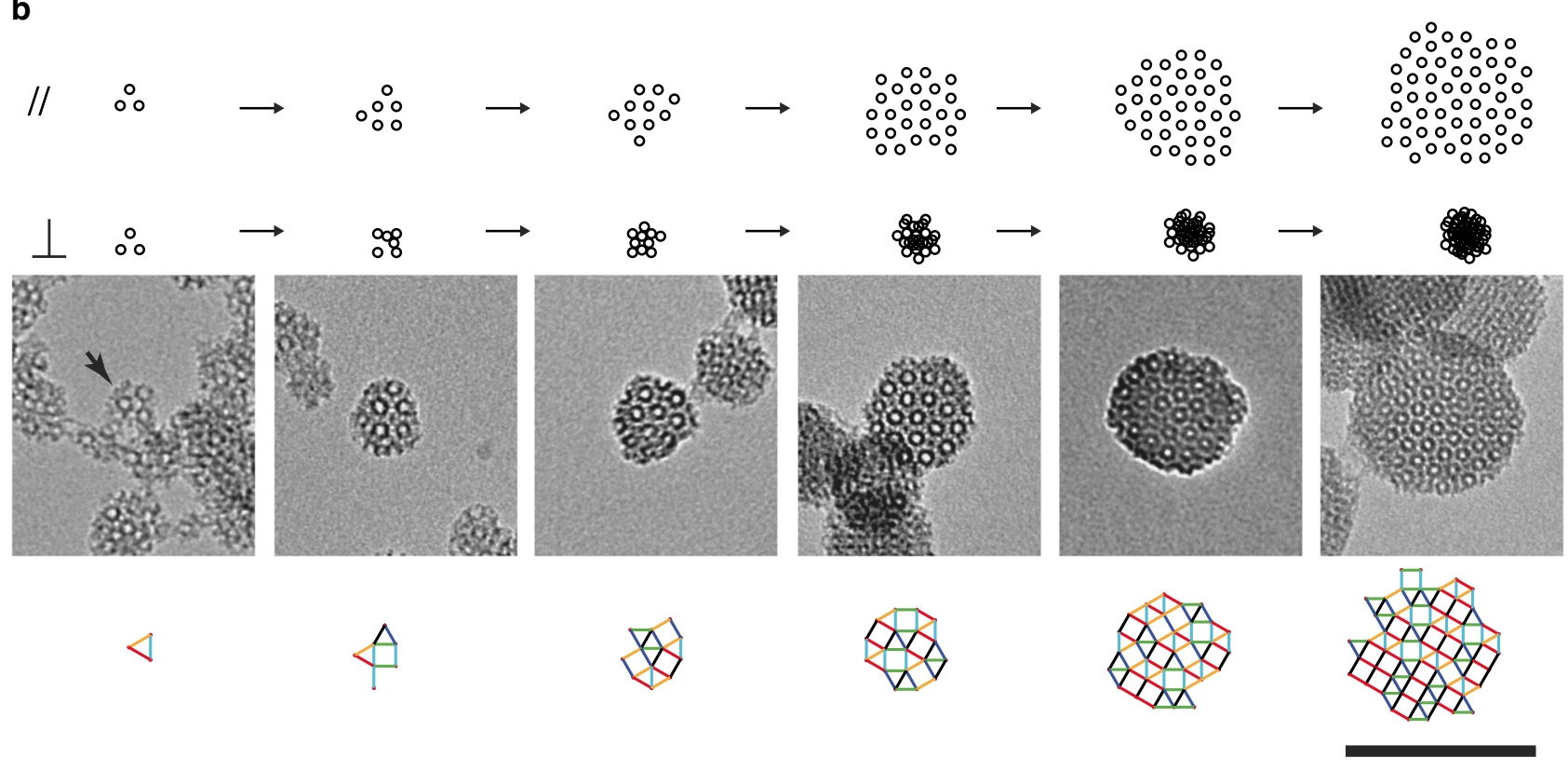

Fig. 6 Comparison of simulated growth trajectories with TMOS/APTMS-derived MSNs. a, b Single-particle growth trajectories of two simulated particles with input triangle/square ratio equal to 0.1 with vertices plotted in both parallel and perpendicular space. Simulated growth stages are compared to experimental TEM images of TMOS/APTMS-derived MSNs and their tilings. Particles were synthesized under varying ammonium hydroxide and mesitylene concentrations as shown in Fig. 2e. The stirring rate was $600 \mathrm{rpm}$ for all syntheses. All images have the same magnification; scale bar is $100 \mathrm{~nm}$ 
Themis 300 operating at $300 \mathrm{kV}$ equipped with a cryo-box and FEI Ceta $16 \mathrm{M}$ camera. More details can be found in the Supplementary Methods.

SAXS patterns were obtained at the G1 station at the Cornell High Energy Synchrotron Source (CHESS) using a $10 \mathrm{keV}$ beam and a sample-to-detector distance of $40 \mathrm{~cm}$. All samples were powders prepared by vacuum drying MSN suspensions after CTAB removal and were imaged soon after drying.

Nitrogen sorption measurements were performed using a Micromeritics ASAP2020 instrument. For each measurement, approximately $10 \mathrm{mg}$ of freshly vacuum-dried powder sample was degassed at room temperature under vacuum overnight prior to the analysis.

Tiling analysis. Tilings were obtained from TEM images using a home-built MATLAB program, where a $10 \%$ tolerance of edge orientation and edge length were allowed and underdeveloped pores on MSNs were not counted. MSNs were excluded from analysis only if particle orientation prevented visualization of the pore structure from TEM images. No additional selection criteria were applied. These tilings were further analyzed using home-built programs to calculate radius of gyration in both parallel and perpendicular spaces. Detailed methods are provided in the Supplementary Methods.

Simulations. An irreversible triangle-square tiling model was developed so that particle growth trajectories could be studied. Simulations were performed employing a self-written MATLAB program. See descriptions in the main text as well as more details in the Supplementary Methods.

Data availability. The data that support the findings of this study are available from the corresponding authors on request.

Received: 11 January 2017 Accepted: 23 June 2017

Published online: 15 August 2017

\section{References}

1. Shechtman, D., Blech, I., Gratias, D. \& Cahn, J. Metallic phase with long-range orientational order and no translational symmetry. Phys. Rev. Lett. 53, 1951-1953 (1984).

2. Förster, S., Meinel, K., Hammer, R., Trautmann, M. \& Widdra, W. Quasicrystalline structure formation in a classical crystalline thin-film system. Nature 502, 215-218 (2013).

3. Zeng, X. et al. Supramolecular dendritic liquid quasicrystals. Nature $\mathbf{4 2 8}$, 157-160 (2004).

4. Hayashida, K., Dotera, T., Takano, A. \& Matsushita, Y. Polymeric quasicrystal: mesoscopic quasicrystalline tiling in A B C star polymers. Phys. Rev. Lett. 98, 195502 (2007).

5. Talapin, D. et al. Quasicrystalline order in self-assembled binary nanoparticle superlattices. Nature 461, 964-967 (2009).

6. Xiao, C., Fujita, N., Miyasaka, K., Sakamoto, Y. \& Terasaki, O. Dodecagonal tiling in mesoporous silica. Nature 487, 349-353 (2012).

7. Huang, M. et al. Selective assemblies of giant tetrahedra via precisely controlled positional interactions. Science 348, 424-428 (2015).

8. Yue, K. et al. Geometry induced sequence of nanoscale Frank-Kasper and quasicrystal mesophases in giant surfactants. Proc. Natl. Acad. Sci. USA 113, 14195-14200 (2016).

9. Gillard, T. M., Lee, S. \& Bates, F. S. Dodecagonal quasicrystalline order in diblock copolymer melt. Proc. Natl. Acad. Sci. USA 113, 5167-5172 (2016).

10. Lee, S., Leighton, C. \& Bates, F. S. Sphericity and symmetry breaking in the formation of Frank-Kasper phases from one component materials. Proc. Natl. Acad. Sci. USA 111, 17723-17731 (2014).

11. Iacovella, C. R., Keys, A. S. \& Glotzer, S. C. Self-assembly of soft-matter quasicrystals and their approximants. Proc. Natl. Acad. Sci. USA 108, 20935-20940 (2011)

12. Van der Linden, M. N., Doye, J. P. K. \& Louis, A. A. Formation of dodecagonal quasicrystals in two-dimensional systems of patchy particles. J. Chem. Phys. 136, 054904 (2012).

13. Joseph, D. \& Elser, V. A model of quasicrystal growth. Phys. Rev. Lett. 79, 1066-1069 (1997).

14. Nagao, K., Inuzuka, T., Nishimoto, K. \& Edagawa, K. Experimental observation of quasicrystal growth. Phys. Rev. Lett. 115, 075501 (2015).

15. Yanagisawa, T., Shimizu, K., Kuroda, K. \& Kato, C. The preparation of alkyltrimethylammonium-kanemite complexes and their conversion to microporous materials. Bull. Chem. Soc. Jpn 63, 988-992 (1990).

16. Kresge, C., Leonowicz, M., Roth, W., Vartuli, J. \& Beck, J. Ordered mesoporous molecular sieves synthesized by a liquid-crystal template mechanism. Nature 359, 710-712 (1992)
17. Hoffman, F., Cornelius, M., Morell, J. \& Fröba, M. Silica-based mesoporous organic-inorganic hybrid materials. Angew. Chem. Int. Ed. 45, 3216-3251 (2006).

18. Sakamoto, Y. et al. Direct imaging of the pores and cages of three-dimensional mesoporous materials. Nature 408, 449-453 (2000).

19. Suteewong, T. et al. Multicompartment mesoporous silica nanoparticles with branched shapes: an epitaxial growth mechanism. Science 340, 337-341 (2013).

20. Suteewong, T. et al. Highly aminated mesoporous silica nanoparticles with cubic pore structure. J. Am. Chem. Soc. 133, 172-175 (2011).

21. Ma, K., Sai, H. \& Wiesner, U. Ultrasmall sub-10 nm near-infrared fluorescent mesoporous silica nanoparticles. J. Am. Chem. Soc. 134, 13180-13183 (2012).

22. Gao, C., Sakamoto, Y., Terasaki, O. \& Che, S. Formation of diverse mesophases templated by a diprotic anionic surfactant. Chem. Eur. J. 14, 11423-11428 (2008).

23. Huo, Q. et al. Generalized synthesis of periodic surfactant/inorganic composite materials. Nature 368, 317-321 (1994).

24. Suteewong, T. et al. Synthesis and formation mechanism of aminated mesoporous silica nanoparticles. Chem. Mater. 24, 3895-3905 (2012).

25. Huh, S., Wiench, J. W., Yoo, J., Pruski, M. \& Lin, V. S. Y. Organic functionalization and morphology control of mesoporous silicas via a cocondensation synthesis method. Chem. Mater. 15, 4247-4256 (2003).

26. Ziherl, P. \& Kamien, R. D. Maximizing entropy by minimizing area: towards a new principle of self-organization. J. Phys. Chem. B 105, 10147-10158 (2001).

27. Ottaviani, M. F. et al. Synthesis of micelle-templated silicas from cetyltrimethylammonium bromide/1,3,5-trimethylbenzene micelles. J. Phys. Chem. B 108, 12123-12129 (2004).

28. Schmidt-Winkel, P. et al. Microemulsion templating of siliceous mesostructured cellular foams with well-defined ultralarge mesopores. Chem. Mater. 12, 686-696 (2000).

29. Blin, J. L. \& Su, B. L. Tailoring pore size of ordered mesoporous silicas using one or two organic auxiliaries as expanders. Langmuir 18, 5303-5308 (2002).

30. Oxborrow, M. \& Henley, C. Random square-triangle tilings: a model for twelvefold-symmetric quasicrystals. Phys. Rev. B 48, 6966-6998 (1993).

31. Ma, K., Mendoza, C., Werner-Zwanziger, U. \& Wiesner, U. Control of ultrasmall sub-10 nm ligand-functionalized fluorescent core-shell silica nanoparticle growth in water. Chem. Mater. 27, 4119-4133 (2015).

32. Firouzi, A. et al. Cooperative organization of inorganic-surfactant and biomimetic assemblies. Science 267, 1138-1143 (1995).

33. Cabane, B. et al. Hiding in plain view: colloidal self-assembly from polydisperse populations. Phys. Rev. Lett. 116, 208001 (2016).

34. Kelley, E. G. et al. Size evolution of highly amphiphilic macromolecular solution assemblies via a distinct bimodal pathway. Nat. Commun. 5, 3599 (2014).

35. Frank, F. C. \& Kasper, J. S. Complex alloy structures regarded as sphere packings. II. Analysis and classification of representative structures. Acta Crystallogr. 12, 483-499 (1959).

36. Ye, X. et al. Quasicrystalline nanocrystal superlattice with partial matching rules. Nat. Mater. 16, 214-219 (2016).

37. Kuo, K. H., Feng, Y. C. \& Chen, H. Growth model of dodecagonal quasicrystal based on correlated tiling of squares and equilateral triangles. Phys. Rev. Lett. 61, 1740-1743 (1988).

38. Stampfli, P. A dodecagonal quasiperiodic lattice in two dimensions. Helv. Phys Acta 59, 1260-1263 (1986)

\section{Acknowledgements}

This work was supported by the U.S. Department of Energy, Office of Science, Basic Energy Sciences, under Award No. DESC0010560. T.K. acknowledges support from the NSF IGERT program (DGE-0966188). K.A.S. and L.F.K. acknowledge support by the Cornell Center for Materials Research (CCMR) with funding from the NSF MRSEC program (DMR-1120296). K.M. and D.Z. thank K. Xie (University of Virginia) for assistance with the development of an automated tiling program. This work made use of the CCMR shared facilities, and the Nanobiotechnology Center shared research facilities at Cornell. The Titan Themis 300 was acquired through NSF-MRI-1429155, with additional support from Cornell University, the Weill Institute and the Kavli Institute at Cornell. This work is based upon research conducted at the Cornell High Energy Synchrotron Source (CHESS), which is supported by the National Science Foundation and the National Institutes of Health/National Institute of General Medical Sciences under NSF Award DMR-1332208.

\section{Author contributions}

Y.S. and K.M. synthesized the materials and performed TEM characterizations. K.M. and T.K. developed the model and performed the simulations. Y.S., K.M., T.K., and D.Z. executed the tiling analysis. H.S. performed the SAXS experiments and their analysis and in-depth characterization of the multicompartment particles. Y.S., K.M., T.K., and H.S. participated in nitrogen sorption experiments and their analysis. K.A.S. and L.F.K. performed cryo-TEM characterization of micelle solutions and image analysis. L.F.K., V.E., and U.W. participated in data interpretation. Y.S., K.M., T.K., V.E., and U.W. 
wrote the manuscript. U.W. supervised the work and was involved in experimental design.

\section{Additional information}

Supplementary Information accompanies this paper at doi:10.1038/s41467-017-00351-8.

Competing interests: The authors declare no competing financial interests.

Reprints and permission information is available online at http://npg.nature.com/ reprintsandpermissions/

Publisher's note: Springer Nature remains neutral with regard to jurisdictional claims in published maps and institutional affiliations. (c) (i) Open Access This article is licensed under a Creative Commons Attribution 4.0 International License, which permits use, sharing, adaptation, distribution and reproduction in any medium or format, as long as you give appropriate credit to the original author(s) and the source, provide a link to the Creative Commons license, and indicate if changes were made. The images or other third party material in this article are included in the article's Creative Commons license, unless indicated otherwise in a credit line to the material. If material is not included in the article's Creative Commons license and your intended use is not permitted by statutory regulation or exceeds the permitted use, you will need to obtain permission directly from the copyright holder. To view a copy of this license, visit http://creativecommons.org/ licenses/by/4.0/.

(C) The Author(s) 2017 